Mathematical Research Letters 6, 697-710 (1999)

\title{
A SMALL UNSTABLE ACTION ON A TREE
}

\section{M.J. Dunwoody}

\section{Introduction}

The theory of groups acting on $\mathbb{R}$-trees was initiated by Tits [15] and by Alperin and Moss [1]. It was developed by Morgan and Shalen [10, 11] and others. A major advance in the theory was the classification by Rips (see [8]) of the finitely generated groups that have free actions on $\mathbb{R}$-trees. Every such group is a free product of free abelian groups and surface groups. This result had been conjectured by Morgan and Shalen.

Let $T$ be an $\mathbb{R}$-tree on which the group $G$ acts (on the left) by isometries. We say that that the action is trivial if for some point $x \in T$ the stabilizer $\operatorname{Fix}(x)=G$. We say that $T$ is minimal if it has no proper $G$-invariant subtree. A nondegenerate (i.e., containing more than one point) subtree $S$ of $T$ is stable if for every nondegenerate subtree $S^{\prime}$ of $S$ we have $\operatorname{Fix}(S)=\operatorname{Fix}\left(S^{\prime}\right)$. A nontrivial action is stable if every nondegenerate subtree of $T$ contains a stable subtree. Bestvina and Feighn [3] have extended the classification of free actions to include all finitely presented groups that have stable minimal actions on $\mathbb{R}$-trees. In particular they show that if $G$ is such a group, then there is an action of $G$ on a simplicial tree $S$ such that if $K<G$ and $K$ fixes an edge of $S$ then $K$ contains a normal subgroup $N$ such that $N$ fixes an arc of $T$ and $K / N$ is abelian. Thus, if in the action of $G$ on $T$ arc stabilizers are small, i.e., they do not contain non-cyclic free subgroups, then edge stabilizers in $S$ are small also.

This paper contains an example of a group $H$ that has a non-trivial, unstable action on an $\mathbb{R}$-tree $T$ with finite cyclic arc stabilizers. It is then shown that there is no non-trivial action of $H$ on a simplicial $\mathbb{R}$-tree with small edge stabilizers. This provides a negative answer to Question D of Shalen [12].

The group $H$ is finitely generated but not finitely presented.

The construction of $T$ is by means of a folding sequence of simplicial trees. The basic folding operations were described by Bestvina and Feighn [2] following an earlier treatment by Stallings [14]. Earlier, Chiswell [4] used a folding operation for graphs of groups in his proof of Grushko's Theorem and Dicks [5] used the folding operation for trees to simplify Chiswell's argument. In [6] I introduced

Received June 12, 1995. Revised November 29, 1999. 
another basic folding move which allows the group acting to be changed. We call this move a vertex morphism.

Let $T, T^{\prime}$ be a $G$-tree and a $G^{\prime}$-tree respectively and let $u \in V T$. A vertex morphism is defined to be a surjective morphism (of graphs) $\phi: T \rightarrow T^{\prime}$ and a surjective homomorphism (also denoted) $\phi: G \rightarrow G^{\prime}$ such that $\phi(g x)=\phi(g) \phi(x)$ for every $g \in G$ and $x \in V T \cup E T$. Clearly $\phi$ induces homomorphisms $\phi_{x}: G_{x} \rightarrow$ $G_{\phi(x)}^{\prime}$ for each $x \in V T \cup E T$. We require that $\phi_{x}$ be an isomorphism for each $x \in E T \cup V T$ unless $x$ is a vertex in the same orbit as $u$. We also require that $\phi$ induce an isomorphism $G \backslash T \rightarrow G^{\prime} \backslash T^{\prime}$.

Associated with the $G$-tree $T$ is a graph of groups $(G(-), X)$ where $X=G \backslash T$, which is illustrated in a Bass Serre diagram. A spanning tree in $G \backslash T$ is lifted to $\mathrm{T}$ and each edge and vertex of $G \backslash T$ is labelled with the corresponding stabilizer in $\mathrm{G}$. If the G-action is not free there is a lot of choice in the way one lifts the spanning tree. Let $v \in V X$, be such that $G(v)=H$ is the stabilizer of the vertex $u$ of $T$. The graph of groups associated with the $G^{\prime}$-tree $T^{\prime}$ is $\left(G^{\prime}(-), X^{\prime}\right)$. Then $\phi$ induces an isomorphism $X \cong X^{\prime}$, so that we can identify $X$ with $X^{\prime}$. We then have $G(x)=G^{\prime}(x)$ unless $x=v$.

Conversely suppose we have a $G$-tree $T$ with corresponding graph of groups $(G(-), X)$ and suppose that $\left(G^{\prime}(-), X\right)$ is a graph of groups such that $G(x)=$ $G^{\prime}(x)$ for every $x \in X$ except for a particular $v \in V X$. If there is a surjective homomorphism $\phi_{v}: G(v) \rightarrow G^{\prime}(v)$ (which has to restrict to the identity on each subgroup of the form $G(e)$, for $e \in E X$, $\iota e=v)$, and $G^{\prime}$ is the fundamental group of this graph of groups (for some maximal subtree of $X$ ), then there is a $G^{\prime}$-tree $T^{\prime}$ and a vertex morphism $\phi: T \rightarrow T^{\prime}$, where $\phi: G \rightarrow G^{\prime}$ is defined in the obvious way.

In our example we will use vertex morphisms as above, subdivision and type I and II Stallings folds. The effect of type I and type II folds is shown in the following Bass Serre diagrams. See [2] for full details. Type I and II folds are the only Stallings folds which apply to tree products of groups, i.e., for a group $G$ with an action on a tree $T$ such that $X=G \backslash T$ is also a tree. The Bass Serre theory gives one a way of describing these folding operations. For a Type I fold, since the edges folded together are in different G-orbits, one can arrange the lift so that that edges of the lifted spanning tree are folded together. This makes for a convenient way of describing the operation in terms of the Bass-Serre diagram.

A different choice of the lifted tree $\tilde{X}$ will change the labelling subgroups by conjugation, though of course the group $G$ remains the same. This change of the lifted tree is used in our construction (illustrated in Figure 2) in which the labels of all the vertices and edges which are joined to a particular vertex $u$ by a path passing through a particular edge $e$ (incident with $u$ ) are conjugated by an element $x^{-1} \in G(u)$. If $\tilde{e}$ is the lift of $e$ included in the original spanning tree $\tilde{X}$, then the new spanning tree will include $x \tilde{e}$. A more general account of 

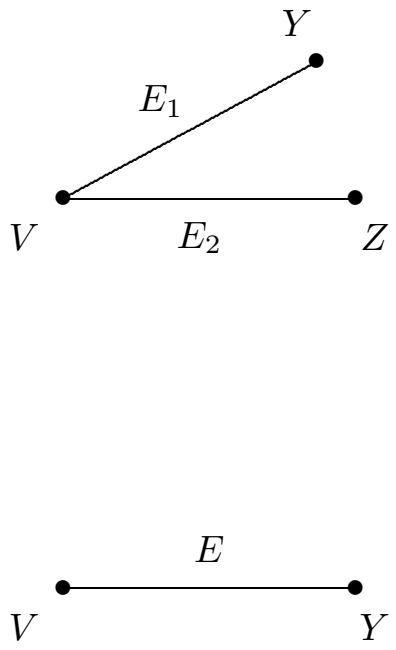

$\stackrel{\text { type } \mathrm{I}}{\Rightarrow}$

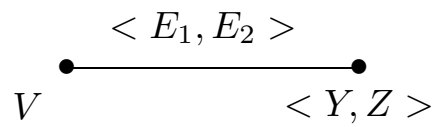

type II

$\Rightarrow$

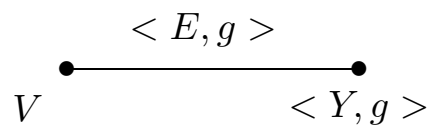

Figure 1

folding sequences is given in [7].

I am grateful to the referee for his care and patience.

\section{Some group theory}

In our construction the alternating groups $A_{n}$ act as building blocks. They are used because if $n$ is composite and odd then $A_{n}$ can be generated by a conjugate pair of elements with order any proper divisor of $n$ that is at least five, and each of the generators is a power of an element of order $n$.

Proposition 1. Let $n=k m$ be odd (so that $k$ and $m$ are both odd), and suppose $m \geq 5$. Let a be a $k$-th power of an $n$-cycle. Then there is an element $b$ of $A_{n}$ such that $a$ and $b^{-1}$ ab generate $A_{n}$.

Proof. We may assume that

$a=(1,2, \ldots, m)(m+1, m+2, \ldots, 2 m) \cdots(m(k-1)+1, m(k-1)+2, \ldots, k m)$.

Let

$$
b=(1,2,3)(m, m+1)(2 m, 2 m+1) \cdots((k-1) m,(k-1) m+1) .
$$

Then

$$
a^{-1} b a=(2,3,4)(1, m+2)(m+1,2 m+2) \cdots((k-2) m+1,(k-1) m+2) .
$$


Put

$$
\begin{aligned}
c= & b^{-1} a^{-1} b a \\
= & (1,4,2, m+2)(m, 2 m+2, m+1)(2 m, 3 m+2,2 m+1) \cdots \\
& \quad((k-2) m,(k-1) m+2,(k-2) m+1)((k-1) m,(k-1) m+1) .
\end{aligned}
$$

(We multiply permutations from left to right). Now $c \in G$, and hence

$$
c^{6}=(1,2)(4, m+2) \in G .
$$

Conjugating by $a$, we have $(2,3)(5, m+3) \in G$. Hence $(1,2,3) \in G$, since $(1,2,3)=((1,2)(4, m+2)(2,3)(5, m+3))^{2}$. Now

$$
\begin{aligned}
& d=a(1,3,2)= \\
& \quad(3,4, \ldots, m)(m+1, m+2, \ldots, 2 m)(m(k-1)+1, m(k-1)+2, \ldots, k m) .
\end{aligned}
$$

Conjugating $(1,2,3)$ by powers of $d$ and $c^{4}$, we get $(1,2, j) \in G$ for $j=3,4, \ldots, n$. It is not hard to show that these elements generate $A_{n}$. Since $m$ is odd, $a$ is an even permutation. Thus $G=A_{n}$. This completes the proof of the Proposition.

Note that, since $k$ is odd, $b \in A_{n}$. There is an $n$-cycle $x$ in $A_{n}$ such that $x^{k}=a$. Thus we see that $A_{n}$ is generated by conjugate $n$-cycles $x, y=b^{-1} x b$ such that $x^{k}, y^{k}$ also generate $G$.

Let $p_{1}<p_{2}<p_{3}<\ldots$ be a sequence of odd primes, $p_{1} \neq 3$. Let

$$
n_{i}=p_{1} p_{2} \ldots p_{i}
$$

Let $K_{i}$ be the alternating group $A_{n}$, where $n=p_{i} p_{i+1} p_{i+2}$. Put $G_{1}=K_{1}$. For $i>1$, let $G_{i}$ be the direct product of $K_{i}$ with a cyclic group of order $n_{i-1}$ generated by the element $k_{i}$. Let $w_{i} \in G_{i}$ be an element which projects to an $n$-cycle in $K_{i}$ and for which $w_{i}^{p_{i} p_{i+1} p_{i+2}}=k_{i}$. Let $w_{i}^{p_{i+2}}=z_{i}$ and $z_{i}^{p_{i+1}}=u_{i}$ so that $u_{i}^{p_{i}}=k_{i}$. By Proposition 1 there exists $b_{i} \in G_{i}$ such that if $v_{i}=b_{i}^{-1} u_{i} b_{i}$, then $G_{i}=\left\langle u_{i}, v_{i}\right\rangle$ and $u_{i}^{p_{i}}=v_{i}^{p_{i}}=k_{i}$. Note that $w_{i}$ has order $n_{i+2}, z_{i}$ has order $n_{i+1}$ and $u_{i}$ has order $n_{i}$.

Suppose now we form the free product with amalgamation

$$
G_{i, i+1}=G_{i} *_{w_{i}=z_{i+1}} G_{i+1} \text {. }
$$

We show that $G_{i, i+1}$ is a direct product $G_{i, i+1}=\left\langle k_{i}\right\rangle \times V_{i, i+1}$, where $V_{i, i+1}$ is the subgroup generated by $K_{i} \cup K_{i+1}$. To see this note that $k_{i}$ commutes with $K_{i}$ and since $k_{i}=u_{i}^{p_{i}}=k_{i+1}^{p_{i}}$, it also commutes with $K_{i+1}$, and hence all of $V_{i, i+1}$. Also, in $G_{i, i+1}, k_{i}=k_{i+1}^{p_{i}}$ and, since $p_{i}$ and $n_{i}$ are coprime, $\left\langle k_{i+1}\right\rangle$ is the direct 
product of $\left\langle k_{i}\right\rangle$ and a cyclic group $C_{i}$ of order $p_{i}$. Thus $G_{i+1}=\left\langle k_{i}\right\rangle \times C_{i} \times K_{i+1}$. The subgroup of $G_{i+1}$ generated by $z_{i+1}$ contains both $k_{i}$ and $k_{i+1}$. Thus there is homomorphism from $G_{i+1}$ on to $\left\langle k_{i}\right\rangle$ which takes $z_{i+1}$ to $k_{i}$ and has kernel $C_{i} \times K_{i+1}$. Since there is a homomorphism from $G_{i}$ on to $\left\langle k_{i}\right\rangle$ which takes $w_{i}$ to $k_{i}$, there is a homomorphism of $G_{i, i+1}$ on to $\left\langle k_{i}\right\rangle$ which contains $V_{i, i+1}$ in its kernel. Note that $C_{i}<\left\langle k_{i+1}\right\rangle<\left\langle w_{i}\right\rangle<G_{i}$, and in fact $C_{i}<K_{i}$, as it is generated by the $n_{i-1}$ 'th power of an element of $G_{i}$. It follows easily that $G_{i, i+1}$ is a direct product as required.

More generally if $i<j$, then

$$
G_{i, j}=G_{i} *_{w_{i}=z_{i+1}} G_{i+1} *_{w_{i+1}=z_{i+2}} * \cdots * w_{j-1}=z_{j} G_{j}
$$

is the direct product of $\left\langle k_{i}\right\rangle$ and the subgroup $V_{i, j}$ generated by $K_{i} \cup K_{i+1} \cup$ $\ldots K_{j}$. For fixed $i$, this can be proved by induction on $j>i$. If $j=i+1$ this has already been proved. If $j>i+1$, then we can assume by the induction hypothesis that $G_{i, j-1}$ is a direct product as indicated. In particular there will be a homomorphism onto $\left\langle k_{i}\right\rangle$ taking $w_{j-1}$ to a generator of the cyclic group. There is also a homomorphism of $G_{j}$ onto $\left\langle k_{i}\right\rangle$ taking $z_{j}$ to the same element. It follows easily that $G_{i, j}$ is a direct product as required.

Note that in $G_{i, j}, w_{j}$ has order $n_{j+2}$ and the subgroup it generates contains the elements $w_{i}, w_{i+1}, \ldots, w_{j-1}$ as well as the direct summand generated by $k_{i}$. If $j \geq i+3$ then $k_{i+3}=w_{i}$. We can write $w_{j}=k_{i}^{\prime} h_{i, j}$, where $k_{i}^{\prime}$ is a generator of $\left\langle k_{i}\right\rangle$ and $h_{i, j} \in V_{i, j}$ has order $p_{i} p_{i+1} p_{i+2} \ldots p_{j+2}$.

Let $X$ be the restricted direct product

$$
X=V_{1,2} \times V_{5,7} \times V_{10,13} \times V_{16,20} \times \cdots .
$$

Thus each factor contains one more $K_{i}$ than the previous one. Let $W$ be the subgroup of $X$ generated by $h_{1,2}, h_{5,7}, h_{10,13}, \ldots$. Then $W$ is a locally cyclic group containing a unique subgroup of order $p_{i}$ for each $i>0$.

Let $i$ be an integer in the set

$$
I=\{1,2,5,6,7,10,11,12,13,16,17,18,19,20, \ldots\} .
$$

Thus $i$ is one of the integers for which there is a monomorphism $K_{i} \rightarrow X$. Note that $I$ contains arbitrarily long finite sequences of successive integers. This property will be important much later on. We now show inductively that we can define monomorphisms $\alpha_{i}: G_{i} \rightarrow X$ in such a way that $\alpha_{i}\left(w_{i}\right)=\alpha_{i+1}\left(z_{i+1}\right)$ if $i, i+1 \in I$ and $\alpha_{i}\left(w_{i}\right)=\alpha_{i+3}\left(k_{i+3}\right)$ if $i, i+3 \in I$. Suppose that $V_{i, j}$ is one of the summands of $X$ and $\alpha_{k}$ has been defined if $k \in I$ and $k<i$. Define $\alpha_{i, j}: G_{i, j} \rightarrow X$ as follows: $\alpha_{1,2}$ is the natural inclusion of $V_{1,2}=G_{1,2}$ in $X$, if $i \geq 5$ set $\alpha_{i, j}\left(k_{i}\right)=\alpha_{i-3}\left(w_{i-3}\right)$ and let $\alpha_{i, j} \mid V_{i, j}$ be the natural inclusion of $V_{i, j}$ in 
$X$. Now define $\alpha_{i}, \ldots, \alpha_{j}$ to be the restrictions of $\alpha_{i, j}$ to $G_{i}, \ldots, G_{j}$ respectively. It can be seen that $\alpha_{i}\left(w_{i}\right)=\alpha_{i+1}\left(z_{i+1}\right)$ if $i, i+1 \in I$ and $\alpha_{i}\left(w_{i}\right)=\alpha_{i+3}\left(k_{i+3}\right)$ if $i, i+3 \in I$, as required. It is also the case that $\alpha_{i}\left(w_{i}\right) \in W$ if $i \in I$.

Let $C_{i, j}$ be a cyclic group of order $p_{i} p_{i+1} \ldots p_{j}$. Let

$$
Y=C_{1,2} \times V_{3,4} \times C_{7,7} \times V_{8,9} \times C_{12,13} \times V_{14,15} \times C_{18,20} \times \cdots .
$$

Here the $V_{i, j}$ summands are chosen to contain precisely the $K_{i}$ subgroups not contained in $X$. The extra cyclic summands are chosen so that $Y$ contains an isomorphic copy of $W$. Thus $h_{3,4} \in V_{3,4}$ has order $p_{3} p_{4} p_{5} p_{6}, h_{8,9} \in V_{8,9}$ has order $p_{8} p_{9} p_{10} p_{11}$, and so the summands $C_{1,2}, C_{7,7}, C_{12,13}$, and so on, fill in the gaps. Let $W^{\prime}$ be the subgroup of $Y$ generated by the elements $h_{3,4}, h_{8,9}, h_{14,15}$ and all the cyclic summands. For each $i>0, i \notin I$ we construct a monomorphism $\beta_{i}: G_{i} \rightarrow Y$. These maps are chosen so that $\beta_{i}\left(w_{i}\right)=\beta_{i+1}\left(z_{i+1}\right)$ if both $i \notin I$, $i+1 \notin I$, also $\beta_{i}\left(w_{i}\right)=\beta_{j}\left(k_{j}\right)^{p_{j-1} p_{j-2} \ldots p_{i+3}}$ if $i, j \notin I, j \geq i+4$, and $\beta_{i}\left(w_{i}\right) \in W^{\prime}$ for each $i \notin I$. Again we use induction on $i$. We know that $G_{3,4} \cong C_{1,2} \times V_{3,4}$, and so we can define $\beta_{3}, \beta_{4}$. Suppose $j, j+1 \notin I$ and $i$ is the largest integer $i<j$, $i \notin I$. Then $Y$ contains a summand $C_{i+3, j-1}$. By induction, we assume that $\beta_{i}\left(w_{i}\right)$ has been defined and that it generates the unique subgroup $W_{i+2}^{\prime}$ of $W^{\prime}$ of order $n_{i+2}$. We know $G_{j, j+1}=\left\langle k_{j}\right\rangle \times V_{j, j+1}$. There is then a monomorphism $G_{j, j+1} \rightarrow X$ which maps $k_{j}$ to a generator of $W_{j-1}^{\prime}=W_{i+2}^{\prime} \times C_{i+3, j-1}$ so that $k_{j}^{p_{i+3} p_{i+4} \ldots p_{j-1}}$ maps to $\beta_{i}\left(w_{i}\right)$, and which is the inclusion on $V_{j, j+1}$. We can now define $\beta_{j}, \beta_{j+1}$ with the required properties, by composing the inclusion maps $G_{j} \rightarrow G_{j, j+1}, G_{j+1} \rightarrow G_{j, j+1}$ with this monomorphism.

Note that the maps $\alpha_{i}$, satisfy the relations $\alpha_{i}\left(w_{i}\right)=\alpha_{j}\left(w_{j}^{p_{j+2} p_{j+1} \ldots p_{i+3}}\right)$, $i, j \in I, i<j$; while the maps $\beta_{j}$ satisfy $\beta_{i}\left(w_{i}\right)=\beta_{j}\left(w_{j}^{p_{j+2} p_{j+1} \cdots p_{i+3}}\right), i, j \notin I$, $i<j$.

Let $L=X *_{W}=W^{\prime} Y$. It follows from the relations stated in the last paragraph that the identification of $W$ and $W^{\prime}$ can be carried out by identifying $\alpha_{i}\left(w_{i}\right)$ with $\beta_{i+1}\left(z_{i+1}\right)$ if $i \in I, i+1 \notin I$.

Proposition 2. The group $L$ has the following properties:-

(i) For each $i$ there is an injective homomorphism $\iota_{i}: G_{i} \rightarrow L$, such that $\iota_{i}\left(w_{i}\right)=\iota_{i+1}\left(z_{i+1}\right)$.

(ii) $X$ is generated by $\bigcup_{i \in I} \operatorname{Im} \iota_{i}$ and $Y$ is generated by $\bigcup_{i \notin I} \operatorname{Im} \iota_{i}$, so that in particular $L$ is generated by the set $\bigcup_{i=1}^{\infty} \operatorname{Im} \iota_{i}$,

(iii) In an action of $L$ on an $\mathbb{R}$-tree with small arc stabilizers, there is a point $x$ fixed by the subgroup $X$ and a (not necessarily distinct) point $y$ fixed by $Y$.

Proof. Define $\iota_{i}: G_{i} \rightarrow L$, so that for $i \in I, \iota_{i}$ is $\alpha_{i}$ composed with the inclusion of $X$ in $L$; while if $i \notin I, \iota_{i}$ is $\beta_{i}$ composed with the inclusion of $Y$ in $L$. Clearly conditions (i) and (ii) are satisfied. 
To show condition (iii) is satisfied, let $T$ be an $\mathbb{R}$-tree with small arc stabilizers. Consider the action of the subgroup $X$. The subgroups $V_{i, j}$ are direct summands of $X$. If one of these summands, say $V$, acted non-trivially on $T$, i.e., there is no vertex fixed by it, then there exists a hyperbolic element $a \in V$ with axis $C_{a}$. Let $U$ be a $V_{i, j}$ summand distinct from $V$. Every element $b \in U$ commutes with $a$, and so $b C_{a}=C_{a}$. But $U$ is a perfect group and has no non-trivial action on a line. Thus $U$ must fix every point of $C_{a}$. But $U$ is not small, since none of the $G_{i, j}$ and hence none of the $V_{i, j}$ is small. Hence we have a contradiction. Thus each $V_{i, j}$ acts trivially on $T$. In fact, each $V_{i, j}$ must fix a unique point of $T$, since $V_{i, j}$ is not small. However the points fixed by distinct $V_{i, j}$ must be the same, since otherwise the summands would not commute. Thus there is a unique point $x$ fixed by $X$. Similarly there is a unique point $y$ fixed by $Y$. This completes the proof of Proposition 2.

We write $\bar{G}_{i}$ for $\iota_{i}\left(G_{i}\right)$, and $\bar{k}_{i}$ for $\iota_{i}\left(k_{i}\right), \bar{w}_{i}$ for $\iota_{i}\left(w_{i}\right)$ and so on. Let $L_{i}$ be the subgroup generated by $\bar{G}_{1} \cup \bar{G}_{2} \cup \ldots \bar{G}_{i-1}, i=2,3, \ldots$ Note that there is a surjective homomorphism $L_{i} * \bar{w}_{i-1}=\bar{z}_{i} \bar{G}_{i} \rightarrow L_{i+1}$, which restricts to the identity on the factors. In $L$ we have the following identities:

$$
\bar{w}_{i-3}=\bar{z}_{i-2}=\bar{u}_{i-1}=\bar{k}_{i} .
$$

\section{The folding sequence}

The construction is by means of a folding sequence. We will define a sequence of simplicial trees $T_{n}$, actions of groups $H_{n}$ on the $T_{n}$, surjective homomorphisms from $H_{n}$ to $H_{n+1}$ and morphisms $T_{n} \rightarrow T_{n+1}$, each of which is a product of elementary folding operations, and so is equivariant in terms of these homomorphisms. It will be shown that the sequence is strongly convergent in the sense of [9]. It follows that there is a limit $\mathbb{R}$-tree which is the limit of this sequence and there is an action of $H$ on $T$ where $H$ is the direct limit of the sequence of homomorphisms $H_{n} \rightarrow H_{n+1}$. Since the homomorphisms are surjective and $H_{2}$ is finitely generated, $H$ is finitely generated.

Consider then the folding sequence of simplicial trees for which the associated graphs of groups are as in Figure 2. The first tree $T_{2}$ in the sequence has one orbit of edges. The corresponding group $\mathrm{H}_{2}$ is a free product with amalgamation $H_{2}=G_{2} * u_{2}=\bar{z}_{1} L_{2}$, which acts on $T_{2}$, and in $T_{2}$ the edge $e$ joining the vertices fixed by $G_{2}$ and $L_{2}$ has length one. One first subdivides each edge of $T_{2}$ by creating a vertex at the midpoint of each edge. Type II folds are then used to enlarge the group of the midpoint so that it becomes a free product with amalgamation of two cyclic groups. There is then a vertex morphism which changes the group associated with this vertex to $G_{3}$. The crucial point about this operation is that the two edge groups become conjugate in the vertex group. Thus we can change our choice of the lift of the spanning tree in the fourth 


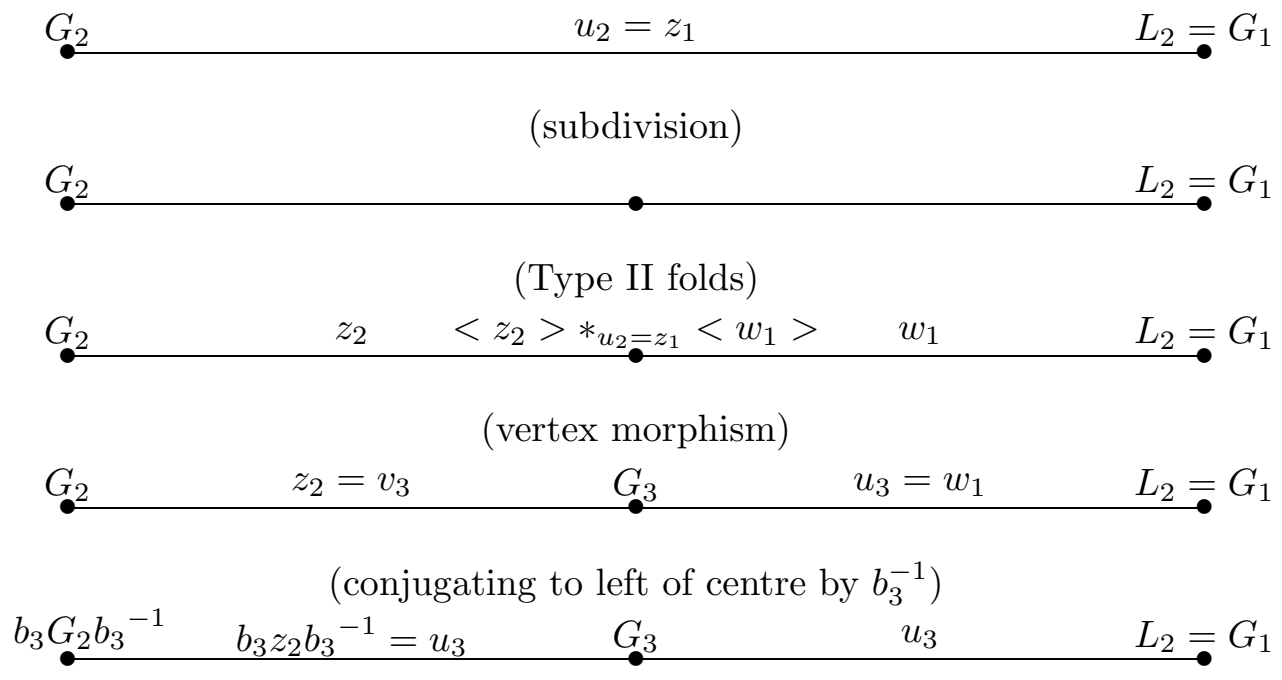

(Type I Fold)

$$
G_{3} \quad u_{3} \quad L_{2} * u_{3} b_{3} G_{2} b_{3}{ }^{-1}
$$

(vertex morphism)

$T_{3}$

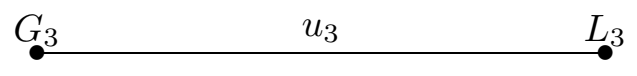

(subdivision and Type II folds)

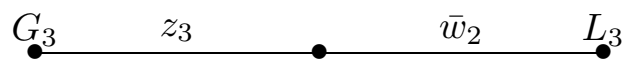

(vertex morphism and conjugating to left of centre)

$$
b_{4} G_{3} b_{4}^{-1} \quad u_{4} \quad G_{4} \quad u_{4}=\bar{w}_{2} \quad L_{3}
$$

(Type I fold and vertex morphism)

$T_{4}$

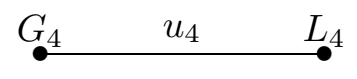

(repeat last three steps infinitely many times)

Figure 2

diagram of the sequence so that both edge stabilizers are generated by $u_{3}$. One can then carry out a Type I fold in which the edges folded together have the same group, and so the resulting edge also has the same group. The fold creates a vertex group on the right which is generated by $b_{3} G_{2} b_{3}^{-1}$ and $L_{2}$. In fact the vertex group on the right is the free product with amalgamation of these two groups in which the amalgamated subgroup is generated by $u_{3}$. This can be seen by examining the action of this new vertex group on the tree associated with the 
previous diagram. For this tree $L_{2}$ and $b_{3} G_{2} b_{3}^{-1}$ act on vertices in distinct orbits. These vertices are separated by two edges, each of which has $\left\langle u_{3}\right\rangle$ as its stabilizer. The subgroup generated by two such vertex groups must be a free product with amalgamation. This group is then changed to $L_{3}$ by a vertex morphism, in which the factor $b_{3} G_{2} b_{3}^{-1}$ is mapped to $\bar{G}_{2}$ by the obvious homomorphism. The tree resulting from these operations is denoted $T_{3}$. Thus $T_{3}$ is an $H_{3}$-tree where $H_{3}=G_{3} * u_{3}=\bar{z}_{2} L_{3}$ and we can repeat the cycle described above. In general $T_{n}, n=2,3 \ldots$, is a subsequence of the sequence of simplicial $\mathbb{R}$-trees shown in Figure 2. Let $H_{i}$ be the group acting on $T_{i}$. Let $\rho_{n}: T_{n} \rightarrow T_{n+1}, n \geq 2$ be the morphism which is the composite of the folds as shown in Figure 2, for $n=2,3,4$. There are two vertex morphisms occurring in each $\rho_{n}$. The first involves the centre vertex. The induced homomorphism on the associated vertex group is

$$
\left\langle z_{2}\right\rangle *_{u_{2}=\bar{z}_{1}}\left\langle\bar{w}_{1}\right\rangle \rightarrow G_{3}, z_{2} \mapsto v_{3}, w_{1} \mapsto u_{3}
$$

for $n=2$, and

$$
\left\langle z_{n}\right\rangle * u_{n}=\bar{z}_{n-1}\left\langle\bar{w}_{n-1}\right\rangle \rightarrow G_{n+1}, z_{n} \mapsto v_{n+1}, \bar{w}_{n-1} \mapsto u_{n+1}
$$

for $n>2$. Note that the orders of elements are such that this homomorphism restricts to an injective map on edge groups. The second vertex morphism involves the right hand vertex. We have seen that there is a surjective homomorphism $L_{n} * \bar{w}_{n-1}=\bar{z}_{n} \bar{G}_{n} \rightarrow L_{n+1}$, which restricts to the identity on the factors. Clearly there is a surjective homomorphism

$$
L_{n} * \bar{w}_{n-1}=b_{n+1} z_{n} b_{n+1}^{-1} b_{n+1} G_{n} b_{n+1}^{-1} \rightarrow L_{n+1},
$$

in which the homomorphism restricts to the identity map on $L_{n}$ and induces the obvious isomorphism $b_{n+1} G_{n} b_{n+1}^{-1} \rightarrow \bar{G}_{n}$ when restricted to $b_{n+1} G_{n} b_{n+1}^{-1}$.

Let $\theta_{n}=\rho_{n} \rho_{n-1} \ldots \rho_{2}: T_{2} \rightarrow T_{n}$. Let $d_{n}$ be the metric in $T_{n}$. Let $x_{2}, y_{2} \in T_{2}$ and put $x_{n}=\theta_{n-1}\left(x_{2}\right), y_{n}=\theta_{n-1}\left(y_{2}\right)$. We show that the non-increasing sequence $\delta_{n}=d_{n}\left(x_{n}, y_{n}\right)$ is eventually constant. It will then follow that there is an $\mathbb{R}$-tree $T$ on which $H$ acts by isometries and the sequence $T_{n}$ together with the composites of the $\rho_{n}$ form a strongly convergent system of simplicial trees. The concept of strong convergence was introduced by Gillet and Shalen [9]. See also Shalen [13]. The definition of $H$ as a direct limit gives rise to natural homomorphisms from the $G_{i}$ and $L_{i}$ to $H$, and these maps are injective. These homomorphisms are induced by the group homomorphisms associated with the morphisms $\rho_{n}$. Thus, for example, we can follow what happens to the group $G_{2}$ as we go down the sequence starting at the fourth diagram. Suppose $G_{2}$ is the stabilizer of the vertex $x$ in the corresponding tree and $L_{2}$ is the stabilizer of the vertex $y$. The lift of the spanning tree is changed so that it includes $b_{3} x$ 
instead of $x$. In the subsequent fold $b_{3} x$ and $y$ are identified. This fold will also identify $x$ with $b_{3}^{-1} y$ and so $G_{2}$ is mapped into the stabilizer of the identified vertex which is generated by $b_{3}^{-1} L_{2} b_{3}$ and $G_{2}$. The next vertex morphism takes this group to $b_{3}^{-1} L_{3} b_{3}$, in particular it takes $G_{2}$ to $b_{3}^{-1} \bar{G}_{2} b_{3}$.

We identify $L_{i}$ with its image in $H$ and we identify $L$ with the union of the subgroups $L_{i}$ in $H$. In an attempt to avoid confusion, we denote the image of $G_{n}$ in $H$ by $\hat{G}_{n}$ and we write $\hat{z}_{i}, \hat{u}_{i}, \hat{b}_{i}$ etc., for the images of the elements $z_{i}, u_{i}, b_{i}$ etc. It follows that $\bar{G}_{n}=\hat{b}_{n+1} \hat{G}_{n} \hat{b}_{n+1}^{-1}$.

We show first that $\theta_{n}$ restricts to an isometry on each edge; it suffices to show that it restrict to an isometry on $e$, which is a representative of the single orbit of edges. Clearly for $\rho_{2}$ the only point of $e$ at which folding can take place is at the midpoint. This will not occur in a vertex morphism since the two halves of $e$ are in different $\mathrm{H}_{2}$-orbits. The Type I fold, which is a factor of $\rho_{2}$, identifies edge pairs (of the appropriate image of $T_{2}$ ) which have the same stabilizer. The two parts of $e$ are mapped to a pair of edges whose stabilizers are $\left\langle u_{3}\right\rangle$ and $\left\langle v_{3}\right\rangle$ and so they are not folded together. A similar argument applies for $\rho_{n}, n>2$ applied to $\theta_{n-1}(e)$.

Consider now the effect of folding on the edges incident with a fixed vertex. In particular consider a vertex $p$ in the tree $T_{n}$ with stabilizer $G_{n}$. There is an incident edge $e$ with stabilizer $\left\langle u_{n}\right\rangle$ for which the other vertex has stabilizer $L_{n}$. The edges of $T_{n}$ incident with $p$ form a single orbit under $G_{n}$, and so every such edge is ge for some $g \in G_{n}$. Let $g e$, he be two such edges. We assume they are distinct which means that $g\left\langle z_{n}\right\rangle=h\left\langle z_{n}\right\rangle$ They will be folded together in the Type II folds in passing from $T_{n}$ to $T_{n+1}$ and from $T_{n+1}$ to $T_{n+2}$ if and only if $g\left\langle w_{n}\right\rangle=h\left\langle w_{n}\right\rangle$. No further folding together of the edges incident with $p$ in $T_{n}$ occurs as a result of the $\rho_{m}, m>n+1$. This is because $\left\langle\bar{w}_{m}\right\rangle \cap \bar{G}_{n}=\left\langle\bar{w}_{n}\right\rangle$ in $L$ and so if $g\left\langle w_{n}\right\rangle \neq h\left\langle w_{n}\right\rangle$, then $\bar{g}$ and $\bar{h}$ lie in different left cosets of $\left\langle\bar{w}_{m}\right\rangle$, for each $m \geq n+1$. A similar argument applies to the vertex with stabilizer $L_{n}$. Thus for any vertex of $T_{n}$ any folding of the edges incident with that vertex in $T_{n}$ which occurs in the whole subsequent folding process must occur for $\rho_{n}$ or $\rho_{n+1}$.

Consider now the segment $\left[x_{n}, y_{n}\right]$. Let $F_{n}$ be the set of points $u$ of the segment at which folding occurs in the composite $\rho_{m} \rho_{m-1} \ldots \rho_{n}$ for some $m>$ $n$. In fact by the above if folding occurs at $u$ then it will occur for $\rho_{n+1} \rho_{n}$. Now $F_{n}$ is a subset of the set $V_{n}$ of vertices of $\left[x_{n}, y_{n}\right]$, since no folding occurs in the interior of edges. The image of $\left[x_{n}, y_{n}\right]$ under $\rho_{n}$ is a finite subtree of $T_{n+1}$ which contains $\left[x_{n+1}, y_{n+1}\right]$. If $v \in V_{n}$ and there is a segment of $\left[x_{n}, y_{n}\right]$ containing $v$ as an interior point which $\rho_{n}$ maps isometrically into $\left[x_{n+1}, y_{n+1}\right]$, then $v \in F_{n}$ implies $\rho_{n}(v) \in F_{n+1}$. Let $u \in F_{n+1}, u \notin \rho_{n}\left(F_{n}\right)$. There are two edges $e, f$ in $\left[x_{n+1}, y_{n+1}\right]$ incident with $u$ and, by the above, there is no segment of $\left[x_{n}, y_{n}\right]$ which maps isometrically to a segment in $e \cup f$, which contains $u$ 
as an interior point. Thus we can find two disjoint segments $s, t$ of $\left[x_{n}, y_{n}\right]$ containing interior points $p, q$ which map to $u$ such that $\rho_{n}(s) \cap\left[x_{n+1}, y_{n+1}\right]$ is a non-trivial segment of $e$ and $\rho_{n}(t) \cap\left[x_{n+1}, y_{n+1}\right]$ is a non-trivial segment of $f$. We can also choose $s, t$ so that they are the smallest distance apart and then $\rho_{n}([p, q]) \cap\left[x_{n+1}, y_{n+1}\right]=\{u\}$. Clearly $[p, q]$ will contain at least one point from $F_{n}$. If it contains exactly one point of $F_{n}$, then it will be the midpoint of $[p, q]$ which is folded in half by $\rho_{n}$. If $[p, q]$ contains more than one point of $F_{n}$, then $F_{n+1}$ will have fewer elements than $F_{n}$. Thus either $F_{n}=\emptyset$ for some $n$, in which case $\left[x_{n}, y_{n}\right]$ is mapped isometrically by all $\rho_{m}, m>n$, or for some $n$ there is a sequence $u_{n}, u_{n+1}, \ldots$, where for $m \geq n, u_{m} \in F_{m}$ and $u_{m}$ is the midpoint of a segment folded in half with endpoints mapped to $u_{m+1}$ by $\rho_{m}$.

In the composite operation $\rho_{n}$ it is only the Type II folds which result in part of the subdivided edge being folded together. We show that if a Type II fold does fold parts of two edges together then no further folding occurs between the remaining parts of the edges. In particular suppose that a Type II fold in $\rho_{2}$ does not act as an isometry on an adjacent edge pair $e, f$. Thus $e, f$ have a common vertex $v$. We assume that $e$ has stabilizers as in the top line of Figure 2. Thus $e$ has stabilizer $\left\langle u_{2}\right\rangle$ and $v$ has stabilizer $G_{1}$ or $G_{2}$. Suppose that $v$ has stabilizer $G_{1}$. The argument for when the stabilizer is $G_{2}$ is similar. Under $\rho_{2}$ both $e$ and $f$ are subdivided, say $e=e_{1} \cup e_{2}, f=f_{1} \cup f_{2}$ and $e_{1}, f_{1}$ have common vertex $v$. Also there exists $w \in\left\langle w_{1}\right\rangle, w \notin\left\langle z_{1}\right\rangle$ which fixes $v$ and $w e=f$. After the Type II fold, $e_{1}, f_{1}$ are folded together. Also $w e_{2}=f_{2}$. If $e_{2}$ and $f_{2}$ were identified in the subsequent vertex morphism then the image of $w$ under the homomorphism $\left\langle z_{2}\right\rangle *_{u_{2}=z_{1}}\left\langle w_{1}\right\rangle \rightarrow G_{3}$ would be in $\left\langle z_{2}\right\rangle$, which is not the case. Also $e_{2}$ and $f_{2}$ are not identified by any subsequent fold, since the image of $w$ in $G_{3}$ is not in $\left\langle z_{3}\right\rangle$ and the image of $w$ in $L_{n}$ lies in $\bar{G}_{3}$ but not in $\bar{G}_{i}$ for $i=4, \ldots, n-1$. Similar arguments apply for Type II folds in any $\rho_{n}$. It follows that the folding at $u_{m}$ in the above sequence cannot be the result of a Type II fold. The only other possibility is that the folding is the result of a vertex morphism. However vertex morphisms fold complete edges together and so there is a bound on the number of vertex morphisms involved in the $\rho_{m}, m>n$ which do not act as an isometry on $\left[x_{n}, y_{n}\right]$. It follows that the sequence $\left(\delta_{n}\right)$ is eventually constant. This means that the sequence $T_{n}$ together with the morphisms which are composites of the $\rho_{n}$ form a strongly convergent system of simplicial trees (see [13], pp. 605). For such a system there is an $\mathbb{R}$-tree $T$ which is the limit of the system. It is easy to define $T$ directly as follows.

Let $T=T_{2} / \sim$, where $x_{2} \sim y_{2}$ if $\theta_{n}\left(x_{2}\right)=\theta_{n}\left(y_{2}\right)$ for $n$ large. From the above, we can make $T$ into a metric space in which

$$
d\left(\left[x_{2}\right],\left[y_{2}\right]\right)=\lim _{n \rightarrow \infty} d_{n}\left(\theta_{n-1}\left(x_{2}\right), \theta_{n-1}\left(y_{2}\right)\right) .
$$

It is clear that $T$ is an $\mathbb{R}$-tree, since the convex closure of any finite set of points 
is the isometric image of a closed, connected subset of $T_{n}$ for $n$ sufficiently large. There is a morphism $\omega_{n}: T_{n} \rightarrow T$, and $\omega_{n}=\omega_{n+1} \rho_{n}$. Recall that $H$ is the direct limit of the sequence of surjective homomorphisms $H_{2} \rightarrow H_{3} \rightarrow \ldots$ We see that $H$ acts by isometries on $T$. There are surjective homomorphisms (also denoted) $\omega_{n}: H_{n} \rightarrow H$. Let $\left(x_{n}\right), x_{n} \in T_{n}$ define a point $x \in T$. Thus $\rho_{n}\left(x_{n}\right)=x_{n+1}$ and $\omega_{n}\left(x_{n}\right)=x$. The homomorphisms $\omega_{n}: H_{n} \rightarrow H$ are injective when restricted to stabilizers. The stabilizer of $x$ is the union of the images of the stabilizers of the $x_{n}, n=2,3, \ldots$ This follows because if $h \in H$ has defining sequence $\left(h_{n}\right)$, then $h x=x$ if and only if $h_{n} x_{n}=x_{n}$ for $n$ large. Each vertex of $T_{n}$ is mapped by $\omega_{n}$ into a single $H$-orbit of points in $T$. This is because the two orbits of vertices of $T_{n}$ are mapped by $\rho_{n}$ into the same orbit of vertices of $T_{n+1}$. Every other point of $T$ has a locally cyclic stabilizer. Thus a finite non-cyclic subgroup of $H$ fixes a unique point of $T$.

We now show that arc stabilizers in $T$ are finite cyclic. It suffices to consider arcs which are contained in the image of the edge $e$ of $T_{2}$, since every arc is covered by finitely many translates of this arc. We identify the image of $e$ in $T$ with the unit interval $[0,1]$. Let $x, y \in e$. Let $m$ be the smallest positive integer for which the open interval $(x, y)$ contains a rational number of the form $k / 2^{m}$. Then $[x, y]$ is stabilized by a conjugate of $u_{m-1}$, since it is contained in the image of an edge of $T_{m-1}$. It is not contained in the image of an edge of $T_{m}$, and from the folding sequence the two halves of an edge of $T_{m-1}$ are stabilized by different conjugates of $u_{m}$. Hence the stabilizer of the arc $[x, y]$ is cyclic of order $n_{m-1}$.

Recall that we identified $L_{i}, i=2,3, \ldots$ and $L$ with subgroups of $H$. The $L_{i}$ form an ascending chain of subgroups of $H$. Let $p$ be the unique point of $T$ stabilized by all of these subgroups. By the above the stabilizer $p$ is in fact the union $L=\cup_{i=2}^{\infty} L_{i}$. Each point of $T$ has stabilizer a conjugate of $L$ or an infinite locally cyclic group. In particular the action of $H$ on $T$ is non-trivial. Since arc stabilizers are finite cyclic, the action is small.

We show now that there is no non-trivial action of $H$ on a simplicial $\mathbb{R}$-tree with small edge stabilizers. Recall that $\omega_{n}\left(G_{n}\right)$ is denoted $\hat{G}_{n}$, and that $\bar{G}_{n}$, which is a subgroup of $L$, is equal to $\hat{b}_{n+1} \hat{G}_{n} \hat{b}_{n+1}^{-1}$. Let $S$ be a simplicial $H$-tree with small edge stabilizers. By Proposition 2 , in the action of the subgroup $L$ on $S$, there is a unique vertex $x$ fixed by $X$, and a unique vertex $y$ fixed by $Y$. For each $n, \hat{G}_{n}$ fixes some vertex $q_{n}$. Note that $\hat{G}_{n+1}=\left\langle\hat{z}_{n}, \hat{b}_{n} \hat{w}_{n-1} \hat{b}_{n}^{-1}\right\rangle<$ $\left\langle\hat{G}_{n-1}, \hat{G}_{n}\right\rangle$. Thus if a vertex is fixed by both $\hat{G}_{n-1}$ and $\hat{G}_{n}$, then it is fixed by all $\hat{G}_{m}, m>n$. Suppose first that $x=y$, so that $L$ fixes $x$. If for any $n, q_{n}=x$ then $H=\left\langle\hat{G}_{n}, L_{n}\right\rangle$ fixes that vertex and the action is trivial. Suppose $q_{n} \neq x$ for all $n$. Choose $n$ and $q_{n}$ so that the geodesic $\left[q_{n}, x\right]$ joining $q_{n}$ and $x$ has the smallest number of edges. Here we use the fact that the action is simplicial. Suppose firstly that this distance is non-zero. Let $y_{n}$ be the vertex of $\left[q_{n}, x\right]$ that is closest 
to $q_{n+1}$. Note that $y_{n}$ lies in both $\left[q_{n}, q_{n+1}\right]$ and $\left[x, q_{n+1}\right]$. Now $\hat{v}_{n+1} \in \hat{G}_{n} \cap \hat{G}_{n+1}$ and $\hat{u}_{n+1} \in L \cap \hat{G}_{n+1}$. Thus $y_{n}$ is stabilized by $\left\langle\hat{u}_{n+1}, \hat{v}_{n+1}\right\rangle=\hat{G}_{n+1}$ and so we can assume $y_{n}=q_{n+1}$. We have a contradiction, unless $q_{n}=q_{n+1}$, since if this is not the case, then $\left[q_{n+1}, x\right]$ has fewer edges than $\left[q_{n}, x\right]$. Thus we may suppose $q_{n}=q_{n+1}=p$ say. In fact we obtain a contradiction unless $q_{m}=p, m \geq n$. Thus $p$ is fixed by every $\hat{G}_{m}, m \geq n$. Hence $p$ is fixed by $\left\langle\hat{b}_{n+1} \hat{G}_{n} \hat{b}_{n+1}^{-1}, \hat{b}_{n+2} \hat{G}_{n+1} \hat{b}_{n+2}^{-1}\right\rangle=\left\langle\bar{G}_{n}, \bar{G}_{n+1}\right\rangle$, which is a subgroup of $L$, and this subgroup is not small. It follows that $p=x$, and we have a contradiction. Suppose then that $x \neq y$. For each $n$, there is a vertex $q_{n}$ fixed by $\hat{G}_{n}$. Again using the fact that the action is simplicial, choose $n$ and $q_{n}$ so that $q_{n}$ has the smallest distance from $[x, y]$. Let $S_{n}$ be the smallest subtree of $S$ containing the vertices $x, y$ and $q_{n}$. Suppose $q_{n+1} \notin S_{n}$. Let $y_{n}$ the vertex of $S_{n}$ that is closest to $q_{n+1}$. Note that $y_{n}$ lies in $\left[q_{n}, q_{n+1}\right],\left[x, q_{n+1}\right]$ and $\left[y, q_{n+1}\right]$. Now $\hat{v}_{n+1}=\hat{z}_{n} \in$ $\hat{G}_{n} \cap \hat{G}_{n+1}$. Also either $x$ or $y$ is stabilized by $\bar{G}_{n}$. But $\hat{u}_{n+1} \in \bar{G}_{n} \cap \hat{G}_{n+1}$ and so it fixes $y_{n}$. Thus $y_{n}$ is stabilized by $\left\langle\hat{u}_{n+1}, \hat{v}_{n+1}\right\rangle=\hat{G}_{n+1}$ and so we can assume $y_{n}=q_{n+1}$. We have a contradiction, unless $q_{n}=q_{n+1}$, since if this is not the case, then $q_{n+1}$ is closer to $[x, y]$ than $q_{n}$ is. Thus we may suppose $q_{n}=q_{n+1}=q_{m}, m \geq n$. But we can choose $m \geq n$ so that $m, m+1 \in I$. Then $\left\langle\hat{b}_{m+1} \hat{G}_{m} \hat{b}_{m+1}^{-1}, \hat{b}_{m+2} \hat{G}_{m+1} \hat{b}_{m+2}^{-1}\right\rangle=\left\langle\bar{G}_{m}, \bar{G}_{m+1}\right\rangle$ is a subgroup of $X$ which also fixes $q_{n}$, and this subgroup is not small. This contradiction means that we can assume $q_{n} \in[x, y]$ for all $n$.

Let $n \in I$. A similar argument to the above shows that there is a vertex of $\left[q_{n}, x\right]$ fixed by $\hat{G}_{n+1}$, i.e., we can choose $q_{n+1} \in\left[q_{n}, x\right]$. Since $I$ contains arbitrarily long sequences of consecutive integers, $q_{n}=q_{n+1}$ for some $n$, so that $q_{n}$ is fixed by $\hat{G}_{m}$ for all $m>n$. It follows easily that neither $\left[q_{n}, x\right]$ nor $\left[q_{n}, y\right]$ can have a small stabilizer. Since at least one of $\left[q_{n}, x\right],\left[q_{n}, y\right]$ is non-trivial, we have a contradiction.

We summarize the above in a theorem.

Theorem. There is a finitely generated group $H$ which acts on an $\mathbb{R}$-tree $T$ so that the action is minimal non-trivial and so that all arc stabilizers are finite cyclic. There is no non-trivial action of $H$ on a simplicial tree with small edge stabilizers.

The group $H$ does have a non-trivial simplicial action on a tree, since

$$
H \cong L *_{J} M,
$$

where $M=\left\langle\hat{G}_{2}, \hat{G}_{3}, \ldots\right\rangle$, and $J=L \cap M=\left\langle\hat{b}_{3} \hat{G}_{2} \hat{b}_{3}^{-1}, \hat{b}_{4} \hat{G}_{3} \hat{b}_{4}^{-1}, \ldots\right\rangle=$ $\left\langle\bar{G}_{2}, \bar{G}_{3}, \ldots\right\rangle$. To see this let $K=L *_{J} M$. Clearly there is a surjective homomorphism $\theta: K \rightarrow H$. However the homomorphism $\omega_{n}: H_{n} \rightarrow H$ factors through $\theta$, since $H_{n} \cong L_{n} *_{u_{n}} G_{n}$. It follows that $\theta$ is an isomorphism.

I think it may be possible to find a similar example which has no non-trivial action on a simplicial tree. 


\section{References}

[1] R.C. Alperin and K.N. Moss, Complete trees for groups with a real-valued length funcition, J. London Math. Soc. (2) 31 (1985), 55-68.

[2] M. Bestvina and M. Feighn, Bounding the complexity of simplicial group actions on trees, Invent. Math. 103 (1991), 449-469.

[3] Stable actions of groups on real trees, Invent. Math. 121 (1995), 287-321.

[4] I.M. Chiswell, The Grushko-Neumann theorem, Proc. London Math. Soc. 33 (1976), 385-400.

[5] W. Dicks, Groups, trees and projective modules, Lecture Notes in Mathematics, 790, Springer-Verlag, Berlin, 1980.

[6] M.J. Dunwoody, Groups acting on protrees, J. London Math. Soc. 56 (1997), 125-136.

[7] , Folding sequences, Geometry and Topology Monographs 1 (I. Rivin, C. Rourke and C. Series, ed.), The Epstein Birthday Schrift, International Press, 1998, pp. 139-158.

[8] D. Gaboriau, G. Levitt, and F. Paulin, Pseudogroups of isometries of $\mathbb{R}$ and Rips' theorem, Israel J. Math. 87 (1994), 403-428.

[9] H. Gillet and P.B. Shalen, Dendrology of groups in low Q-ranks, J. Differential Geom. 32 (1990), 605-712.

[10] J.W. Morgan and P.B. Shalen, Valuations, trees, and degenerations of hyperbolic structures, I, Ann. of Math. (2) 120 (1984), 401-476.

[11] Valuations, trees, and degenerations of hyperbolic structures, II, Ann. of Math. (2) 127 (1988), 403-465.

[12] P.B. Shalen, Dendrology of groups: an introduction, Essays in Group Theory, MSRI Publ., Math. Sci. Res. Inst. Publ., 8, Springer-Verlag, New York-Berlin, 1987, pp. 265-319.

[13] Dendrology and its applications, Group Theory from a Geometrical Viewpoint (Trieste, 1990), World Sci. Publishing, River Edge, NJ, 1991, pp. 543-616.

[14] J.R. Stallings, Topology of finite graphs, Invent. Math. 71 (1983), 551-565.

[15] J. Tits, A theorem of Lie-Kolchin for trees, Contributions to algebra (a collection of papers dedicated to Ellis Kolchin), Academic Press, New York, 1977, pp. 377-388.

Faculty of Mathematical Studies, University of Southampton, Southampton, SO17 1BJ.

E-mail address: mjd@maths.soton.ac.uk 